\title{
Editorial
}

\section{Who Dropped the Ball on L-dopa? A Patient's Lament}

\author{
Jon Palfreman ${ }^{* 1}$ \\ School of Journalism and Communication, University of Oregon, OR, USA
}

How's the war on Parkinson's disease going? That depends on whether you're a researcher, clinician, or patient. Researchers can claim that they are making spectacular progress in understanding the disease. Clinicians can argue that they manage the symptoms better than at any time in history. If you're a patient like me, however, then the honest truth is that the war isn't going very well.

The Holy Grail of biomedical research is an intervention that slows, stops, or reverses a disease-a so-called disease-modifying procedure. Over the last decade or so, Phase 3 clinical trials of diseasemodifying therapies for Parkinson's disease have reported almost universally disappointing results. When tested under placebo-controlled conditions, the three main disease-modifying approaches-protecting neurons (with drugs like selegiline and rasagiline), reviving neurons (with neurotrophic factors like GDNF and neurturin), and replacing neurons (with neural grafts) - have so far failed to deliver. There are many possible reasons for the failures. It may be that the trial designs were flawed. Or it might be that the interventions didn't work because the disease pathology in the patients being studied was already too advanced to modify. A landmark paper by Jeff Kordower et al., for example, reported that within 5 years of being diagnosed, Parkinson's disease patients have virtually no functioning dopamine nerve endings left.

If the dream of a transformative disease-modifying therapy still lies a decade or more in the future, what

\footnotetext{
*Correspondence to: Jon Palfreman, School of Journalism and Communication, University of Oregon, OR, USA. E-mail: jpalfreman@pfgmedia.com.

${ }^{1}$ Social Media Editor, Journal of Parkinson's Disease.
}

about progress developing better ways of delivering L-dopa more continuously to mitigate motor complications? A major advance in L-dopa delivery that eliminated-or at least minimized-on-off fluctuations, freezing of gait, and dyskinesias would likely improve patients' lives significantly. You might think that since L-dopa was first used in the 1960s-a period during which we have gone to the moon, invented the Internet, and sequenced the human genome-investigators might have cracked this puzzle. But there has been staggeringly little progress. And I think I know at least part of the reason why. Many researchers simply don't find the quest to solve the levodopa delivery problem very exciting and neuroscientists and clinicians are itching to move on to new frontiers. Consider the two big trends currently in vogue. One popular among neuroscientists is the quest to develop really early interventions, which target, for example, alpha-synuclein aggregation or mitochondrial failure. In this model, Parkinson's disease might be stopped years before it manifests its cardinal symptoms. If it pans out, such a spectacular advance-the ultimate disease-modifying intervention-would, of course, benefit future generations but not necessarily those who currently have the disease. A second theme, popular among many neurologists, is the push to move the clinical focus away from the traditionally dopamine-centered motor problems like tremor and rigidity. As the distinguished neuroscientist and clinician Jon Stoessl wrote recently, "we have reasonably good treatments for the motor manifestations of Parkinson's disease... . The challenge of Parkinson's disease is the management of non-motor problems, many of which have a non-dopaminergic basis." 
But hang on a minute. While it's certainly true that attention should be given to the plethora of disabling non-motor symptoms like depression, sleep disorders, constipation, dementia etc., it seems premature to close the book on better L-dopa delivery. For the millions of Parkinson's disease patients in the world, solving this problem would be life changing.

To be fair, the challenge of getting L-dopa into the brain is not trivial. Here's what happens when a patient swallows a standard yellow 25/100 carbdidopalevodopa tablet. The pill passes down the esophagus, and enters the stomach. Within about 15-30 minutes, it reaches the upper GI tract-a small eighteen-inch section of intestine that includes the duodenum and jejunum. This section is a key departure terminal for molecules destined for the brain. Molecules like levodopa-which are identical to naturally occurring substances in our bodies such as amino acids-are readily absorbed along this short stretch of gut. They are taken up by the large neutral amino acid transporter, a kind of conveyor belt that carries a vast cargo of chemicals exiting the stomach en route to the body's tissues. The $100 \mathrm{mg}$ of levodopa in the pill vies for space on this conveyor belt with whatever else is exiting the stomach-e.g. amino acids digested from proteins that were part of breakfast.

As the conveyor passes through the body — visiting skeletal muscle, liver, kidney etc-enzymes attack its molecular cargo ${ }^{2}$. These enzymes prematurely convert most of the levodopa into dopamine, which can't cross the blood brain barrier. In an attempt to limit the loss, manufacturers wrap the $100 \mathrm{mg}$ of levodopa with 25 $\mathrm{mg}$ of powerful decarboxylase enzyme blockers (carbidopa or benserazide ${ }^{3}$ ).

Over the next 2-3 hours, even with the protection of the decarboxylase inhibitor, only about $5-10 \%$ of the levodopa in the pill actually makes it to the brain, crosses the blood-brain barrier, and is converted into dopamine. And that dopamine isn't delivered steadily to the neuronal receptors, but unevenly, starting with small amounts, building to a maximum, and falling to almost nothing over the course of about three hours. Patients report experiencing this rise and fall, detecting when their medicine kicks in, when it peaks and when it wears off. As the disease progresses, the patient might have an increasingly more difficult time coping with this uneven delivery. Advanced patients end up

\footnotetext{
${ }^{2}$ The two most important ones are L-aromatic amino acid decarboxylase (AADC) and catechol-O-methytransferase (COMT). Something like $70 \%$ of the levodopa will be broken down by AADC and another $10 \%$ by COMT.

${ }^{3}$ Carbidopa and benserazide inhibit AADC.
}

having to take six or seven pills a day just to keep moving.

Over the years, neuroscientists and pharmaceutical companies have tried to deliver L-dopa more evenly and continuously. They made a version of carbidopa-levodopa that is released more slowly in the GI tract (Sinemet CR). They tried combining carbidopa-levodopa with another enzyme inhibitor called entacapone (Stalevo). They tried blocking the breakdown of dopamine in the brain (using selegiline and rasagiline) and they even attempted to replace L-dopa with drugs called dopaminergic agonists (pramipexole, ropinirole, and rotigotine), molecules designed to mimic dopamine. None of these significantly improved the patient experience.

The effort failed says Henry Ford Hospital Professor Peter LeWitt, because the products were based on "a lot of half-hearted pharmaceutical development, with poor design, and not enough clinical research." Take the Sinemet CR, for example. Once it passed beyond the critical 18 inches of upper GI tract where the absorption took place, its slowly releasing drug load was simply wasted. Stalevo might have been effective if the entacapone enzyme blocker had been delivered to the GI tract before levodopa. It wasn't. In studies, Stalevo reduced off time but increased dyskinesias. Dopaminergic agonists, for most patients, turned out to be pale imitations of levodopa and had their own unpleasant side effects. MAO-B inhibitors like selegiline and rasagiline had a mild therapeutic effect but were hardly game changers. But LeWitt says that unfortunately, "when they discovered such problems, the drug companies didn't drop the project and then go on to something better, they finished up marketing the product regardless of what the data showed."

Fortunately, not everyone has abandoned the quest for continuous dopamine delivery. Indeed, the Michael J Fox Foundation for Parkinson's Research has spent millions supporting several innovative symptommodifying ideas ${ }^{4}$. I want to mention two of them. California-based Depomed embeds the carbidopalevodopa in a special polymer designed to swell up on contact with the stomach's gastric juice. Rather than passing into the ileum and large intestine, the swollen polymer lodges in the stomach, strategically positioned just above the critical absorbing region of upper GI

\footnotetext{
${ }^{4}$ In addition to Depomed and Neuroderm's technologies (discussed here), MJFFPR has supported Civitas, which produces an inhalable levodopa fast-acting "rescue" therapy, Intec's "accordion pill" (another gastric-retention strategy for more continuous dopaminergic effect), and Cynapsus, which makes an oral apomorphine rescue technology.
} 
tract. Over 8-10 hours the drug-polymer hybrid erodes, delivering a steady dose of levodopa. This ingenious idea (a "gastric retention" platform called AcuForm) has already been FDA-approved to deliver antibiotics, diabetes drugs, and pain medications and the evidence is that it can do the same for L-dopa. In Phase 1 and Phase 2 trials, patients receiving two daily doses of Depomed's product displayed much more constant levodopa concentrations-avoiding the peaks and troughs - and experienced fewer motor complications, than patients getting five doses of immediate-release carbidopa-levodopa.

An Israeli Company, Neuroderm, has developed an even more revolutionary approach - to bypass the entire GI tract altogether and deliver a liquid formulation of carbidopa-levodopa subcutaneously. In a brilliant piece of chemistry, Neuroderm scientists figured out how to produce a stable, concentrated liquid form of carbidopa-levodopa that can be practically delivered into the blood stream-a challenge that had defeated chemists for half a century. Using a "pump-patch," or belt pump, similar to those used by Type-I diabetes patients, Neuroderm's platform delivers carbidopa-levodopa continuously over 24-hours.

While such technologies are works in progress, ${ }^{5}$ it's all very exciting. Peter LeWitt (who is a research and scientific consultant for Depomed and Neuroderm) says, "the near constant levels produced by these products are remarkable... and comparable with expensive, invasive technologies like Duodopa." (Duodopa, a technology that continuously infuses carbidopa-levodopa gel through a surgically fitted tube in the jejunum, is approved in Europe and Canada, but not yet in the US.).

So, if Depomed and Neuroderm products offer simple, non-invasive ways of achieving the long sought after goal of steady L-dopa delivery, why haven't we heard more about them? One disturbing possibility appears to be that despite their potential importance to patients, big pharma finds them commercially unattractive. To get these products to market involves not only Phase 1 and 2 studies (which the companies have undertaken), but also two expensive Phase 3 clinical trials and a one-year-long FDA New Drug Application review. So, typically, small companies like Depomed and Neuroderm need financial investment, often in the form of a pharmaceutical industry partner, to afford this final stage. Remarkably, LeWitt says finding a partner may not be easy. " The big question is will

\footnotetext{
${ }^{5}$ Other companies developing dopamine delivery products include Intec and Xenoport.
}

the pharmaceutical industry take the bait and develop these therapies based on what is very hard scientific evidence."

I asked Jerry Callahan-Vice President for Business Development at Van Andel Research Institute and the founder of two venture capital firms specializing in the life sciences-why giants like Pfizer, Glaxo Smith Kline, or Roche aren't lining up to partner with Depomed and Neuroderm? Since levodopa is an established drug taken by millions of people, the risks of adverse effects are likely vanishingly small. But what about commercial risks? What he told me was sobering. When deciding on new investments in the biotech industry, pharmaceutical companies typically ask several questions. Is there an unmet need? Does the new product meet the unmet need? How big is the potential market? Will the payers (Medicare, Medicaid, private insurance) agree to premium prices?

A life-threatening condition, like an incurable form of say cancer or heart disease, is an unmet need for vast numbers of people. A new cancer drug that extends life can probably, therefore, command a premium price from payers. Novartis charges $\$ 76,000$ a year, for example, for its drug Gleevec. But, says Callahan, "Parkinson's is not like cancer, it's not like heart disease. Patients don't typically die from Parkinsons' disease. A person with Parkinsons' disease is usually in their 60s, has lived a long life, and many are already retired. It's bad, but it's not life-threatening."

The commercial risk analysis goes like this. Since the proposed new products won't extend lives but will rather simply improve the lives of people who already have access to L-dopa, payers like Medicare might not approve premium pricing (say $\$ 10,000$ a year compared to the currently $\$ 1,000$ per year spent on Sinemet) for this "convenience." "Now people who know Parkinsons' disease," says Michael J Fox Foundation CEO Todd Sherer, " know that it's hardly a matter of convenience. Advanced patients need improved delivery because they keep going on and off, and they keep freezing up, and they're dyskinetic ... A steady continuous dose of L-dopa would allow people to be more productive, to keep their jobs, creating real life impacts." Over and above helping advanced patients with few options, some neuroscientists believe that in theory treating early stage patients from the outset with continuous dopamine delivery might avoid motor complications altogether. Such a prospect if true would radically improve a newly diagnosed patient's trajectory.

Callahan cited another possible pharmaceutical company concern—numbers. Depomed had no trouble 
finding a partner when they used their AcuForm platform to deliver the pain drug gabapentin. That's no big surprise, says Callahan. "Pain is a monster market. Compared with 1.5 million Parkinsons' disease sufferers there are something like 75 to 80 million people taking pain medication for things like back pain and cancer, by comparison people with Parkinsons' disease have a weak voice."

I'd never really thought of Parkinson's disease patients having a weak voice. But when you consider everything competing for attention, then it behooves the Parkinson's disease community to up its game. Our voice would be much stronger, of course, if neurologists and neuroscientists were out there educating the payers in Medicare and private insurance of the importance of better L-dopa delivery products-from significant increases in "on" time, to reducing freezing of gait, falls, and dyskinesias. But neuroscientists I have spoken to appear to find L-dopa delivery boring. As Sherer puts it, "If I'm a neuroscientist, do I want to work on some fascinating new mechanism like prions-you know things that are on the cutting edge-or do I want to spend time on reformulating a drug that's been around for 50 years?" What about neurologists? Sherer says that once these products reached the clinic, "neurologists would likely want them in their toolkit, but they don't focus on basic research." In other words, most neurologists may be unaware these potentially life changing products are languishing in the drug pipeline.

What makes the apparent lack of interest in and knowledge about new dopamine delivery technologies especially baffling — given the arguments of unmet need, patient numbers, and payment-is the commercial success of deep brain stimulation and Duodopa. How did the biomedical establishment command premium reimbursements for these two highly invasive Parkinson's disease therapies that are only approved for a small segment of the Parkinson's market? Deep Brain Stimulation, which costs about $\$ 100,000$ for the extensive surgery, implants and programming appointments, is covered by Medicare, Medicaid, and Private Insurance. And Abbvie Pharmaceuticals' Duodopa-a $\$ 60,000$ a year intervention requiring about two weeks in the hospital to attach an intestinal pump and then daily cassettes with the carbidopa-levodopa gel-is covered in Europe and Canada and, likely, soon in the US.

If an effective Depomed or Neuroderm-type product can, in principle, do the same job as these invasive, risky procedures at a fraction of the cost then why isn't there a rush to get them to market? To quote Todd Sherer, "that's a very good question." 